\title{
Caught in time
}

The detection of cancer at an early stage in its development can be life-saving. With research efforts under way to find better methods to detect minuscule tumours, Laura Spinney finds out how near some of these cancer 'biomarkers' are to the clinic.

W

hen it comes to halting cancer, nobody disputes the value of early

detection. Dr George Papanicolaou's smear test, which measures changes in the cervical lining, is a testament to the importance of screening. Since 1950, when it was introduced, the Pap smear has reduced mortality due to cervical cancer by $70 \%$ in developed countries. About half of lung-cancer patients whose cancer is caught while it is still restricted to the lung survive. Nearly all of those diagnosed after it has spread die. (See graphic, opposite.)

Yet for all its promise, early detection remains the poor relation of cancer treatment. In 2005, the US National Cancer Institute (NCI) spent around $8 \%$ of its research budget on detection and diagnosis, compared with $22 \%$ on treatment. Britain's National Cancer Research Institute, a collaboration of the country's major cancer-research funders, spent $9 \%$ of its 2004 budget on early detection, diagnosis and prognosis, versus $19 \%$ on treatment.

\section{Seeing the signs}

Nevertheless, the technology needed to detect cancers at the very earliest stages is within sight. Advances in mass spectroscopy and in the detection of mutant DNA, for example, are now making it possible to pick up very low levels of tell-tale molecules. Meanwhile, medical-imaging technology is improving our ability to peer inside the body (see 'Powers of detection', overleaf). Ultimately, scientists hope to devise straightforward, non-invasive tests, such as screening blood samples, to pick up on the earliest signs of problems - perhaps even before cells become fully cancerous.

As a first step, scientists are hunting for effective 'biomarkers' - DNA or proteins that act as indicators of normal or pathological biological processes that could be used to screen for the presence of a cancer. But a big challenge facing biomarker hunters is that blood is a complex soup of molecules containing everything from 
THE VALUE OF EARLY SCREENING

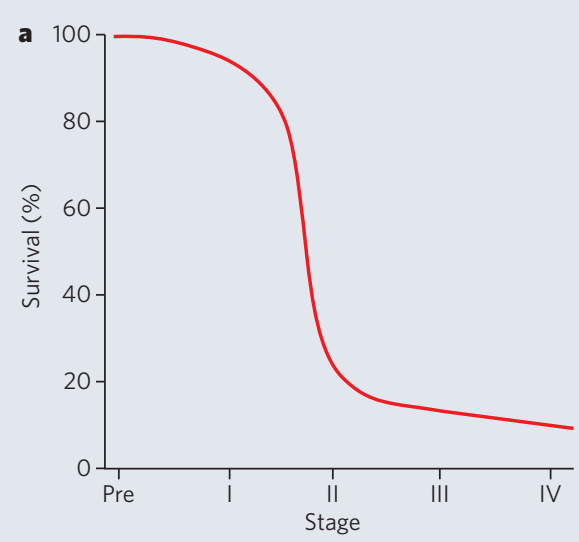

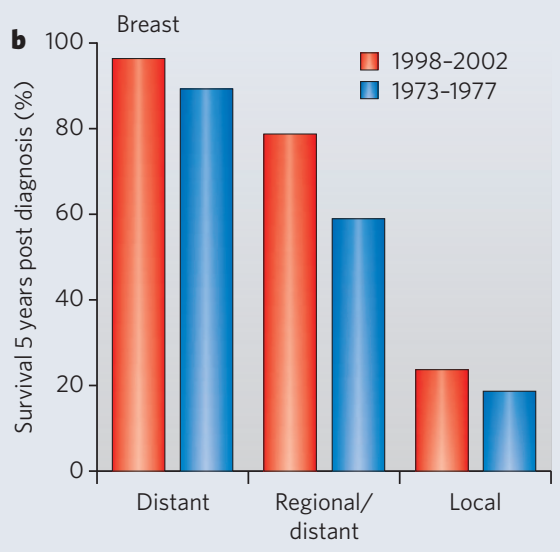

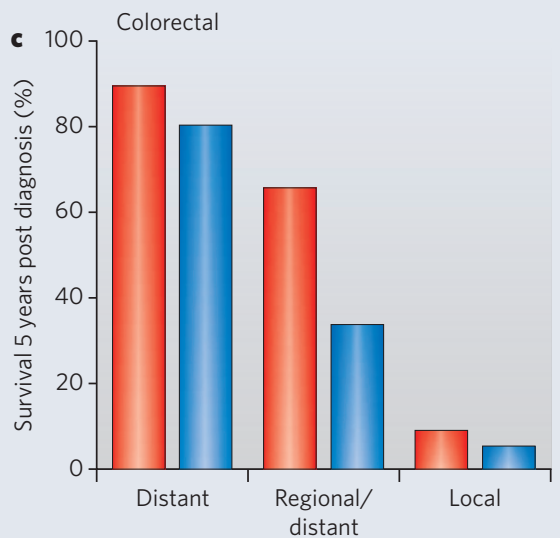

a, Catch a tumour before it becomes malignant (pre-malignant stage) or while it is still small and locally confined (stage I) and the chances of a cure are high. They drop sharply once the cancer spreads to local tissues and lymph nodes (II-II) or other organs (IV). b, c, Despite improvements in treatments since the 1970s, patients still have a greater chance of surviving if cancers are detected when they are locally confined (local) and not spread to neighbouring tissue (regional) or other organs (distant).

tumour and are circulating in the bloodstream; the second is to search for an immune response to the tumour. The host immune system may not be able to destroy the tumour cells, but it probably recognizes them as problem cells, and Hanash wants to harness that raised antibody response as a diagnostic tool. "We're finding that some circulating proteins are inducing an immune response," he says. "We're also finding antibodies to circulating proteins."

Hanash's biomarkers are just some of many that scientists have identified. Underpinning all this work is the understanding that using a single biomarker to diagnose a cancer is unlikely to suffice. "We're all agreed now that to rely on one marker to make a diagnosis is not going to work," says Hanash. No single biomarker can detect a given cancer with $100 \%$ sensitivity (meaning that all diseased subjects would test positive) and $100 \%$ specificity (with all healthy subjects testing negative). Panels of biomarkers with different individual sensitivities and specificities are therefore needed.

A team led by David Sidransky at Johns Hopkins University School of Medicine in Baltimore, Maryland, for example, is trying to find ways of improving the accuracy of the blood tests used to detect a protein called prostate-specific antigen, or PSA. PSA is produced by prostate cancers, but can also result from benign prostrate enlargement or inflammation. Sidransky's team has identified an alteration to a gene called GSTP1 that is unique to prostate cancer cells. The hope is that men with high levels of PSA in their blood could be referred for a biopsy to test for this genetic alteration, to reduce the number of false positives that result from relying on PSA levels alone ${ }^{2}$.

To become fully cancerous, cells go through a complex, stepwise progression from normal cells to diseased ones. Scientists argue ${ }^{3}$ that, for optimal early detection, researchers need to find biomarkers targeted to a stage in this progression where changes can be detected with the highest sensitivity and specificity. That may be a 'pre-malignant' phase, when cells begin to behave abnormally, but have not yet developed all the characteristics of tumour cells.

One example of where this approach is being applied is in research on the common and sometimes crippling gynaecological condition endometriosis, where tissue that normally lines the womb appears on other pelvic organs. Women with endometriosis have a higher risk of developing a particular type of ovarian cancer and last year, Daniela Dinulescu, now at Brigham and Women's Hospital, Harvard Medical School, discovered genetic pathways underlying the progression from endometriosis to cancer in mice ${ }^{4}$. This raises the possibility that a test could one day pinpoint early genetic changes in women. In separate work, Dinulescu is collaborating with Hanash to validate around 20 biomarkers for early ovarian cancer.

\section{Risk assessment}

As researchers rush to assemble biomarker panels, the limiting factor to early detection is becoming the ability to detect these biomarkers in ever smaller quantities. Last year, Bert Vogelstein of Johns Hopkins University and the Howard Hughes

Medical Institute (HHMI) in Chevy Chase, Maryland, showed for the first time that it is possible to detect trace amounts of mutated DNA against a noisy background of unmutated DNA, in a simple blood sample ${ }^{5}$. He adapted a technique called the polymerase chain reaction, or PCR, to detect fragments of a mutated gene called adenomatous poly-

Medical imaging technology will soon be able to detect minuscule tumours. posis coli (APC) in the blood of patients with advanced colorectal cancer. PCR allows researchers to selectively amplify a particular stretch of DNA using an enzyme, which can start work on very small quantities of DNA. Vogelstein's team also detected mutant APC molecules in more than $60 \%$ of patients with early, curable colorectal cancer; these were circulating in the patients' blood in extremely low quantities.

Bioengineer Steve Quake of the HHMI and Stanford University thinks that this is just the beginning. "The fundamental limit of detecting single molecules has been reached in many cases of scientific interest, so in principle, this should be applicable to cancer detection as well," he says.

Given that there is no such thing as a single magic marker for any cancer, Quake feels that the ideal way to detect early disease would be to sequence and so identify all the hundreds of thousands of molecules in a blood sample. "As crazy as it sounds, this is something that is coming within reach of the new 


\section{Powers of detection}

Many scientists working on the early detection of cancer hope to find molecules, or biomarkers, in a person's blood that betray the presence of a tumour at the earliest stages of its development. But a blood test will simply tell you that a tumour exists, not where it is.

To pinpoint a tumour's location, researchers are developing medical-imaging technology that is much more sensitive than conventional mammography or computed tomography (CT) scans and is able to pick up tiny tumours.

The key to determining a tumour's location is to inject a compound or probe, visible to imaging machines, into the bloodstream that seeks out a particular biomarker in the tumour. The compound will then accumulate in the tumour, making it light up on scans. With conventional positron emission tomography (PET) scans, where the probe is radioactive, there are limits to how much of it you can safely introduce into a person. But with newer techniques, such as optical (pictured below) or ultrasound imaging, scientists can inject more of the probe to boost the signal, as the probes, for example, antibodies attached to fluorescent chemicals, are less toxic.

Plus, says Sam Gambhir, director of Stanford University's Molecular Imaging Program, the properties used to assess the signal, such as wavelength, scatter and absorption, simply allow you to see more.

Gambhir says it is currently possible to see down to the level of thousands of cells in animal models such as mice. In humans, the increased complexity and depth of our tissues means the limit is closer to millions of cells. Seeing a single cell in humans would depend on developing strategies with much higher signal amplification and very low noise.

"Nothing we have right now would allow that," he says, "But it is certainly feasible that in humans we could get down to thousands of cells. The imaging community is working on that."

A $1 \mathrm{~mm}^{3}$ tumour contains about 3 million cells; if scientists could get down to thousands of cells, they could detect tumours around $0.1 \mathrm{~mm}^{3}$ in size. Most researchers think that detecting tumours in the range of $0.5 \mathrm{~mm}^{3}$ to $1 \mathrm{~mm}^{3}$ is achievable within the next five to ten years, says Gambhir.

In part, he adds, such improvements depend on selecting the right biomarkers. With the USbased Canary Foundation, a nonprofit organization dedicated to the early detection of cancer, Gambhir has brought together members of the imaging community with those working on biomarkers to ensure the right ones are developed. It's an exciting collaboration. "Molecular imaging is really pushing the envelope of detection," he says. L.S. sequencing technologies," he says, "If you have many markers of somewhat weak utility, the product of the probabilities of all those markers can be very powerful."

But despite all the effort going into biomarker discovery, very few have so far been turned into diagnostic tests. Hartwell says this is because validating them is a slow process. A year from now, he expects that the consortium of seven US research institutions that he leads in the search for breast-cancer biomarkers will have assembled 100 candidates. But they will then have to develop sensitive tests for those markers and only if the tests perform well in distinguishing diseased from healthy individuals, can they apply them to larger populations to measure their sensitivity and specificity.

Sudhir Srivastava, who heads the NCI's Cancer Biomarkers Research Group, says another factor delaying test development is that for the past 30 years, biomarker discovery has been driven largely by scientists doing basic research; such researchers may be less aware of which markers are likely to be most useful clinically. To try to change that, the NCI has brought together dozens of cancer-research institutions into an Early Detection Research Network (EDRN), which already funds a lot of research on detection, including Hanash's lung-cancer work. Bringing biomarkers to clinical application is one of the EDRN's main objectives. "This programme is attempting to strike a balance between discovery and validation," he says. "It brings clinical and basic scientists together, so that discovery is clinically driven."

The speed with which early-detection tests reach the clinic will also depend partly on how much of their development is taken up by pharmaceutical companies. Some fear that, because single biomarkers will turn out to be of limited value, drug firms will steer clear of detection and stick to treatment. Hartwell disagrees. "Uniformly now, all the pharmaceutical companies want biomarkers for their particular drug, to know whether the drug is effective or not," he says. "The other place that this whole field could move into is drugs for prevention." As patients identified as high risk are likely to be taking preventive drugs for a lifetime, this could be a lucrative area for the drug industry.

\section{From bench to bedside}

Drug companies are acutely aware of the potential in this area, agrees Brian Holloway, director of preclinical studies on Iressa (the drug that's used to treat lung cancer), at AstraZeneca in Macclesfield, UK. They are also keen to find ways of targeting their treatments more effectively, he adds. "There is no cancer drug that works for everybody, so drug companies will

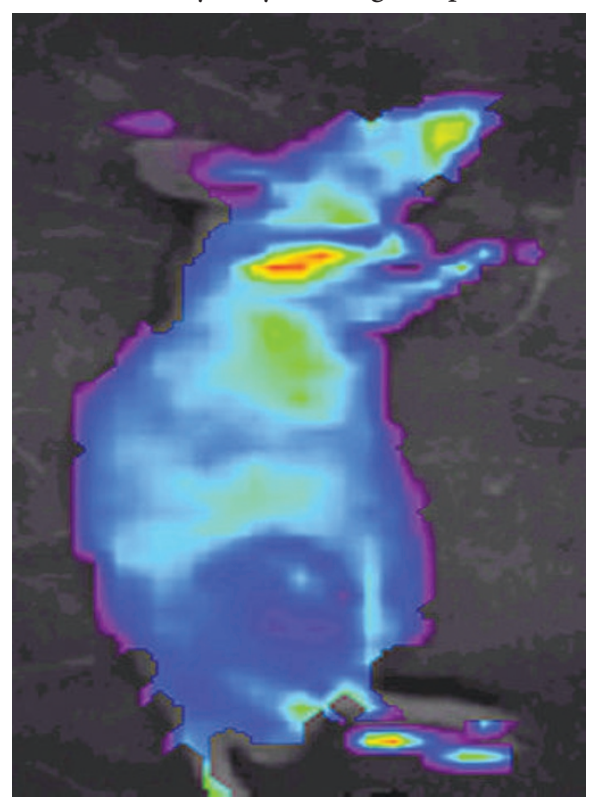

The imaging techniques used to show up tumours (top red spot) are getting increasingly sensitive. look more and more for biomarkers to select the populations that will benefit from a given drug," he says.

As promising as early detection sounds, it does have its problems. For one thing, simply being able to detect the presence of a tumour may not give doctors enough information to treat a patient appropriately. Michael Pollak, director of the Program of Cancer Prevention at McGill University in Montreal, Canada, points out that at least half the world's people older than 60 have cancerous growths, most of which are non-aggressive and will not cause disease. "Early detection of all the lesions would result in a huge industry of medical interventions, most of which would be unnecessary, but a few of which would be life-saving," he says. "Early detection must be able to distinguish incidental from dangerous cancers - a much more difficult job."

And healthcare providers will still have to be convinced that early-detection tests are worthwhile at the population level, something that Richard Sullivan, head of clinical programmes at Cancer Research UK, says is notoriously hard to prove. It involves calculating the cost-effectiveness of the screening test, which in turn requires detailed information on the cost of screening, the long-term costs of cancer care, the sensitivity and specificity of the screening test and its impact on survival. But following Dr Papanicolaou's example, more screening programmes will almost certainly be established in years to come.

Laura Spinney is a science writer based in London and Paris.

1. Gao, W. M. et al. BMC Cancer 5, 110 (2005).

2. Jerónimo, C. et al. J. Natl. Cancer Inst. 93, 1747-1752 (2001).

3. Pollak, M. N. \& Foulkes, W. D. Nature Rev. Cancer 3, 297-303 (2003).

4. Dinulescu, D. M. et al. Nature Med. 11, 63-70 (2005)

5. Diehl, F. et al. Proc. Natl Acad. Sci. USA 102, 16368-16373 (2005). 